\title{
ELECTROMAGNETIC SCATTERING FOR DIELECTRICS: ITERATIVE METHODS FOR SOLVING BOUNDARY INTEGRAL EQUATIONS
}

\author{
B. BIELEFELD, Y. DENG, J. GLIMM, F. TANGERMAN, AND J. S. ASVESTAS
}

\author{
(Communicated by David Sharp)
}

\begin{abstract}
We consider multigrid iterative methods for the solution of electromagnetic scattering for dielectric materials. We show convergence of the iteration using coarse grids which are two to four times coarser in each dimension than the fine grid. These results allow a significant increase in problem size and solution speed, for a given hardware configuration. We report in particular on the solution of scattering problems which require the solution of 31,000 equations on the fine grid, using the direct solution of 3,500 double precision equations on the coarse grid, and project the ability to solve significantly larger systems using larger machines or an out-of-core capability.
\end{abstract}

\section{INTRODUCTION}

Scattering theory, i.e., the relation between a geometrical shape and the waves reflected from it, is important for a number of problems, namely: nondestructive evaluation, sonar, particle accelerator design, radar, seismic exploration, antenna design, and the spacing of devices and wires on a computer chip to avoid interference. When the object and the media are characterized by sharp boundaries and piecewise constant material properties, the appropriate linear wave equations can be integrated using Green's functions, giving rise to boundary integral equations. Although this step reduces the dimensionality of the problem by one, the resulting boundary integral equations are still a formidable challenge to solve numerically at a desired level of detail.

The solution of discretized boundary integral equations requires the inversion of a large full matrix. The two central problems are storage and speed. For $n$ equations, memory requirements are $O\left(n^{2}\right)$ and the speed of the matrix inversion is $O\left(n^{3}\right)$, by LU methods. Of these two restrictions, memory is a more fundamental limit. The record result is the solution of 75,000 equations on the Intel Delta with 512 processors [4-6]. Time is split between the $O\left(n^{2}\right)$

Received by the editors February 5, 1993.

1991 Mathematics Subject Classification. Primary 45F99, 78-08, 15A06; Secondary 65N55.

This project is supported partially by the Applied Mathematics Subprogram of the U.S. Department of Energy DE-FG02-90ER25084 and by the Mathematical Sciences Institute of Cornell University under subcontract to the University at Stony Brook. The third author was also supported by ARO contract number DAAL03-91-C-0027, and the National Science Foundation, grant DMS-9201581. 
matrix evaluation and the $O\left(n^{3}\right)$ matrix solve. Further improvement, and the ability to solve larger systems, requires improving both of these steps.

The purpose of this paper is to report our results in overcoming these obstacles in the context of electromagnetic scattering. We show that optimized matrix fill operations allow evaluation of matrix elements as needed, thus reducing storage. The optimized matrix fill also reduces the solution time by nearly $50 \%$.

Iterative inversion [2] has been proposed to overcome the $O\left(n^{3}\right)$ asymptotics. In this method, an approximate solution on a coarse grid is used to accelerate the iterative inversion of the fine grid equations. Using powerful parallel computers, we are able to refine and test this method. Our results are mixed. If material parameters are not extremely different across an interface, we find dramatic improvements in the speed and in the number of equations which can be solved. We have no difficulty with 31,000 complex equations in double precision on a machine 16 times smaller than the Intel Delta and apparently could solve significantly larger systems. We point out that for extremely dissimilar materials, the iterative method generally has no value, and LU inversion is preferred. This is the case, for instance, if the object is a metallic conductor. Then the boundary integral equations are not always well-posed due to the phenomenon of electromagnetic resonances. The iterative method is much more sensitive to these resonances than is the LU inversion.

The fine grid mesh size $h_{f}$ has to be taken small enough to ensure sufficient accuracy of the solution to the discrete equation. An approximate determination
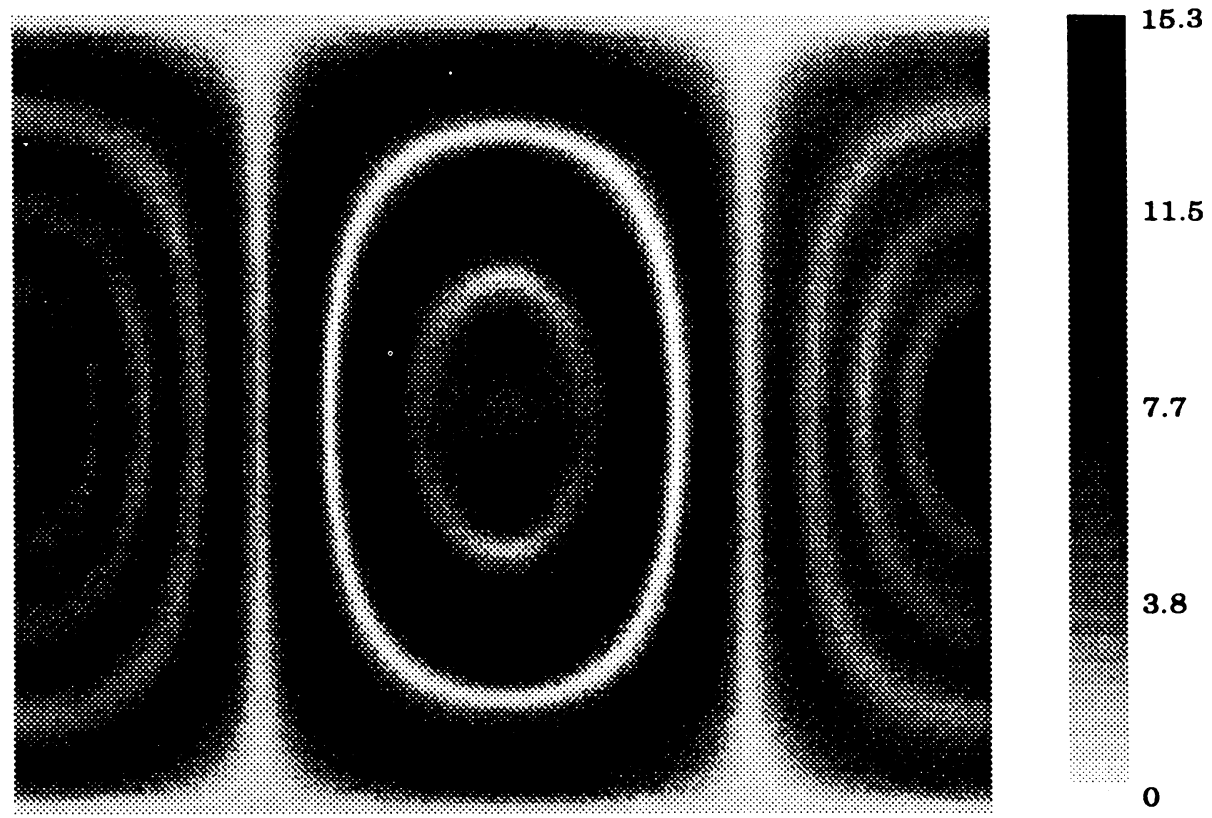

Figure 1. The bistatic radar cross-section [3] indicated by the gray scale for a polystyrene sphere with diameter to inner wavelength ratio $D / \lambda_{\text {in }}=4.9 \quad(k a=$ 15.3). This figure maps spherical coordinates $(\theta, \phi)$ into Cartesian coordinates $(X, Y)$. 
of $h_{f}$ as a function of the incoming wave length $\lambda$ and, in the case of dielectrics, of the relative permittivity $\varepsilon_{r}$ of the object gives

$$
h_{f} \sim \frac{1}{10} \frac{\lambda}{\sqrt{\left|\varepsilon_{r}\right|}} \text { for dielectrics. }
$$

The quantity $\lambda_{\text {in }}=\lambda / \sqrt{\left|\varepsilon_{r}\right|}$ is the wavelength in the body, i.e., the inner wave length.

Our findings are very interesting for dielectrics. Multigrid methods are mathematically appropriate and effective for dielectrics such as plastics and epoxy resins, because the permittivity $\varepsilon$ satisfies $\varepsilon=O(1)$. The main result of this paper is that convergence of the iterative multigrid method can be obtained using a coarse grid with 2.5 to 5 coarse meshes per inner wavelength, yielding the required accuracy within ten iterations. If $n$ denotes the number of equations obtained from the fine grid, then the number of coarse grid equations is on the order of $n / 4^{2}$ to $n / 2^{2}$. This yields a gain with respect to the LU decomposition by a factor of $2^{6}$ to $4^{6}$ in speed and $2^{4}$ to $4^{4}$ in storage. In order to stay within reasonable use of computer resources, our tests used up to 3,500 coarse grid equations and up to 31,000 fine grid equations.

Figure 1 shows the bistatic radar cross-section for a polystyrene sphere, in polar coordinates, with diameter to inner wavelength ratio $D / \lambda_{\text {in }}=4.9 \quad(k a=$ 15.3) .

\section{NUMERICAL METHODS}

The boundary integral equations for electromagnetic scattering from a penetrable homogeneous object have the form $[1,3] L u=v$, where $v$ (respectively, $u$ ) is a vector field (respectively, a pair of vector fields) tangent to the surface $S$. We extend such vector fields to a neighborhood of $S$ by parallel transport in the directions normal to $S$. We discretize these equations through a triangulation of the surface $S$, using a Glisson-basis [7] and dual elements.

A triangulation of $S$ defines a piecewise linear approximation $S^{\prime}$ to $S$ whose triangular faces are determined by the vertices in $S$. If the mesh-size is small enough, $S^{\prime}$ will be close to $S$.

Consider any edge $e$ in $S^{\prime}$ and its two adjacent triangles. This configuration is called the diamond associated with $e$. Name one of the triangles the positive triangle $\Delta(e)^{+}$and the other the negative triangle $\Delta(e)^{-}$. Define the vertex $v(e)^{ \pm}$as the vertex of $\Delta(e)^{ \pm}$which is not on $e$. As in [7] we associate to each edge $e$ a piecewise linear vector field $f_{e}$ tangent to $S^{\prime}$ and supported in the diamond associated to $e$ :

$$
f_{e}(x)= \begin{cases} \pm \frac{1}{2 \operatorname{area}\left(\Delta(e)^{ \pm}\right)}\left(x-v(e)^{ \pm}\right), & x \in \Delta(e)^{ \pm} \\ 0, & x \text { elsewhere }\end{cases}
$$

$f_{e}$ has discontinuities at the edges of the diamond associated with $e$. It is parallel to the edges different from $e$ and the integral of its divergence vanishes. Denote by $V\left(S^{\prime}\right)$ the vector space spanned by such vector fields. These Glisson vector fields are not orthogonal with respect to the standard inner product on tangent vector fields.

We now define a projection map from vector fields tangent to $S$ to $V\left(S^{\prime}\right)$. The main idea is that of "normal flux" through an oriented edge. We first discuss this in the smooth case: given an oriented curve $\gamma$ in $S$ and a tangent vector 
field $f$ to $S$, define

$$
\gamma \bullet v=\int_{\gamma} \operatorname{det}|\dot{\gamma}(s), v(\gamma(s)), n(\gamma(s))| d s .
$$

Here $s$ denotes an arc-length parameter and $n$ denotes the outward pointing unit normal vector to $S$. If $v$ is tangent to $\gamma, \gamma \bullet v$ vanishes. For vector fields $v$ on $S^{\prime}$ which have discontinuities along the edges of $S^{\prime}$ but are continuous on each of the faces, we define for an oriented edge $e$ :

$$
e \bullet v=\frac{1}{2}\left[\int_{e} \operatorname{det}\left|\frac{e}{\|e\|}, v^{+}(e(s)), n^{+}\right| d s+\int_{e} \operatorname{det}\left|\frac{e}{\|e\|}, v^{-}(e(s)), n^{-}\right| d s\right] \text {. }
$$

Here $\|e\|$ denotes the length of $e, v^{ \pm}$denotes the limit of $v$ on $e$ for the triangle $\Delta(e)^{ \pm}$, and $n^{ \pm}$denotes the unit outward pointing normal vector to $\Delta(e)^{ \pm}$. The edges $e$ are oriented and the Glisson vector fields $f_{e}$ are normalized so that

$$
e \cdot f_{e}=+1
$$

Moreover, for every edge $e^{\prime}$ different from $e$

$$
e^{\prime} \bullet f_{e}=0, \quad e^{\prime} \neq e .
$$

Define the projection $\pi_{S^{\prime}}$ of a vector field $v$ on $S^{\prime}$ as

$$
\pi_{S^{\prime}}(v)=\sum_{\text {edges } e}(e \bullet v) f_{e} .
$$

We finally obtain as a discretized equation $L^{\prime} u^{\prime}=v^{\prime}$. Here $L^{\prime}=\pi_{S^{\prime}} \cdot L$ and $v^{\prime}=\pi_{S^{\prime}}(v)$. This equation can be written in matrix form as $\left[L_{i, j}^{\prime}\right]=$ $\left[e_{i} \bullet L^{\prime}\left(f_{e_{j}}\right)\right]$. Since the elements $e$ are dual to the vectors $f_{e}$, this matrix $\left[L_{i, j}^{\prime}\right]$ is the matrix for the operator $L^{\prime}: V\left(S^{\prime}\right) \rightarrow V\left(S^{\prime}\right)$ with respect to the Glisson basis.

2.1. Mesh-refinement. Consider a coarse triangulation $S_{c}$ of $S$. The refinements we consider in this paper are uniform refinement by a fixed integer $p$. Given an integer $p$, divide each of the edges of $S_{c}$ in equal $p$ pieces. Triangles are now subdivided in $p^{2}$ small, congruent, triangles by drawing lines parallel to the edges through the subdivision of the other two edges. The fine triangulation $S_{f}$ of $S$ is obtained by projecting the new vertices normally to $S$.

Given any two triangulations $S_{f}$ and $S_{c}$ we define the injection map $\imath: V\left(S_{c}\right)$ $\rightarrow V\left(S_{f}\right)$ and the projection map $\pi: V\left(S_{f}\right) \rightarrow V\left(S_{c}\right)$ as

$$
l=\pi_{S_{f}} \cdot \mathscr{I}, \quad \pi=\pi_{S_{c}} \cdot \mathscr{I}
$$

where $\mathscr{I}$ denotes a fixed reasonable extension of vector fields. Also

$$
L_{f}=\pi_{S_{f}} \cdot L, \quad L_{c}=\pi_{S_{c}} \cdot L, \quad v_{f}=\pi_{S_{f}}(v) .
$$

2.2. The iterative method. The equation to be solved iteratively is $L_{f} u=v_{f}$. Consider the following approximate inverse to the matrix $L_{f}$ :

$$
M=l\left(L_{c}^{-1}-I\right) \pi+I,
$$


where the matrix $L_{c}^{-1}$ is computed by LU-decomposition. Define the sequence $u_{n}$ recursively as

$$
\begin{aligned}
u_{0} & =M v_{f}, \\
u_{n+1} & =u_{n}+M\left(v_{f}-L_{f} u_{n}\right) .
\end{aligned}
$$

The convergence of this algorithm is well understood if the original operator $L$ has a bounded inverse and is of the form $L=I+K$ with $K$ a compact operator, provided the coarse mesh-size is fine enough and the triangulation is quasi-uniform (uniformly controlled aspect-ratios). The boundary integral equations of the second kind for electromagnetic scattering from dielectrics [1] can be written in this form and have this invertibility property.

This algorithm is a simplification of the two-step iteration method found in [2]. The latter converges twice as fast at the expense of twice as much computation.

2.3. Numerical evaluation. The computation of individual matrix-coefficients involves the integration of functions related to certain Green's functions [1] over the triangles from both of the grids.

Since the size of these triangles are in constant proportion to the wavelength, we can obtain a given accuracy with a fixed number of integration points per triangle. On the coarse grid we used 7 point Gaussian quadrature while we used 1 point Gaussian quadrature (barycenter) on the fine grid. When the size of the coarse grid matrix exceeded 2000, single precision LU decomposition proved to be inaccurate and we had to use double precision in the LU decomposition. We point out that such sizes table-lookup for the computation of the integrands occurring in the entries of the matrix is far more efficient than evaluation.

\section{Parallelization}

In order to perform large experiments, a code, Maxwell3D, has been written and parallelized for a class of distributed-memory MIMD architectures such as the Intel iPSC/860 and TMC CM-5. The code has been run on the 32-node iPSC/860 with $16 \mathrm{MB}$ of memory per node and $2 \mathrm{~GB}$ of disk space (with two I/O nodes).

There are three major steps in the parallelization: (1) creation of the system of linear equations, $L_{f} u=v_{f} ;(2)$ solution of the coarse grid system of linear equations (parallel LU-decomposition [8]); and (3) parallel iterations on two grids on-the-fly. The main idea for all three steps is the technique of row partition. All processors expand their coarse grid equations with the same magnification factor, which ensures perfect load balance. After expansion, the fine grid equations overflow the system memory. We compute the fine grid matrix elements on-the-fly, discarding data that are not presently needed and re-computing when necessary.

We have achieved better than $80 \%$ parallel efficiency for up to 32 processors. The largest problem, 31,000 complex equations, reported in this paper took 20 hours to solve on a 32-node iPSC/860. It is interesting to note that a similar calculation using purely LU decomposition methods for 48,672 equations done on the Intel Delta [5] with 512 compute processors took a similar amount of time, 19.74 hours. 


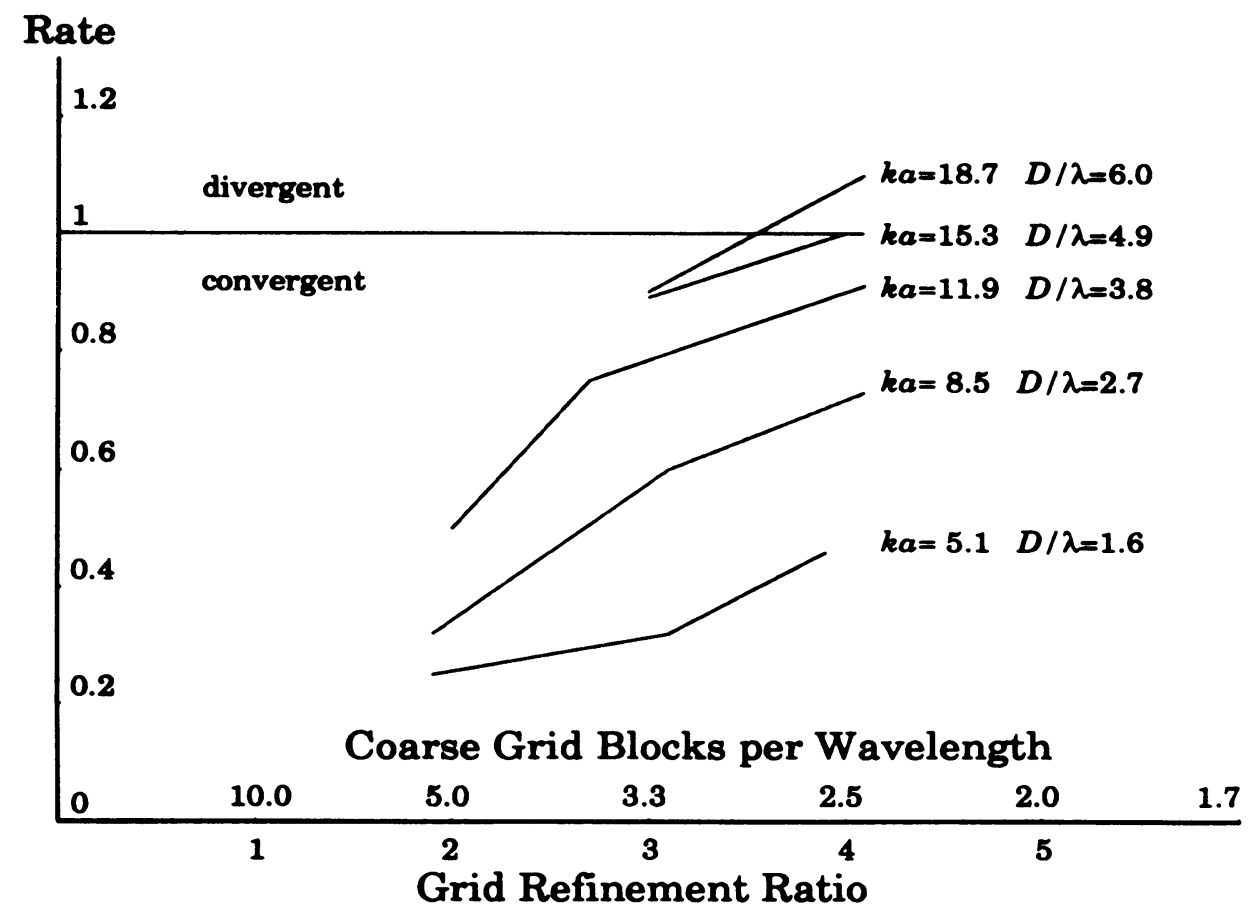

FIGURE 2. The convergence rate of the scheme vs. the coarse-grid mesh blocks per wavelength. In the experiments, a polystyrene sphere is used. $D$ is the diameter of the sphere, $a$ is its radius, $\lambda_{\text {in }}$ is the inner wavelength, and $k$ is the wave number. Thus $k a$ and $D / \lambda$ provide different but equivalent measures of the dimensionless problem size. Note: in this figure, some data points lie on noninteger refinement ratio points. This is because the $\lambda /$ (fine grid mesh-size) is not exactly 10 .

\section{Results}

Figure 2 depicts our results. We use a polystyrene sphere $\left(\varepsilon_{r}=2.5\right)$. In the figure $D$ is the diameter of the sphere, $a$ is its radius, and $\lambda_{\text {in }}$ is the inner wavelength.

If $n$ is the number of iterates, the error is

$$
\left\|u_{\infty}-u_{n}\right\| \approx C r^{n}
$$

where $r$ is the convergence rate of the algorithm. If $r<1$, the algorithm converges. The line $r=1$, separating convergence from divergence, is indicated in Figure 2.

Using 5 coarse meshes per inner wavelength gives a factor of $2^{2}=4$ savings in memory and converges quite well, but our computer ran out of memory for $D / \lambda_{\text {in }}>4$. Using 3.3 coarse meshes per inner wavelength gives a factor of $3^{2}=9$ savings in memory. This resolution converged up to $D / \lambda_{\text {in }}=6$, which was the largest we could fit in the computer. Using 2.5 coarse meshes per inner wavelength gives a savings of $4^{2}=16$ in memory but converges only up to $D / \lambda_{\text {in }}=3.8$. For $D / \lambda_{\text {in }}>4.9$ the rate was either bigger than one or so close 
to one that too many iterates were required for convergence. The solution of 31,000 equations was accomplished in 20 hours using all 32 nodes.

\section{CONCLUSION}

In this paper we have shown that multigrid iterative methods provide striking advantages in the memory requirement as well as execution time for the solution of large electromagnetic scattering problems for dielectrics.

The mesh-size of the fine grid is dictated by accuracy requirements. In this paper we used a 1.5 digit accuracy requirement in the solution, leading to the approximate formula 1. A general feature of these iterative methods is that if one wants higher accuracy, the fine mesh-size must be smaller while the coarse mesh-size can stay the same with the same rate of convergence. Therefore, the more accuracy required, the better the method works.

The as-yet-unexplained aspect of our results is the deterioration in the convergence rate as the dimensionless object size $\left(D / \lambda_{\text {in }}\right)$ increases.

Additional work is needed to allow multigrid methods to be useful for the prediction of scattering from a wider range of materials.

\section{ACKNOWLEDGMENTS}

We thank R. Goldrick and R. Melnik from the Grumman Corporate Research Center, J. Rogers from the Polytechnic University in Brooklyn, and E. Stasic and D. Tao from SUNY for helpful discussions.

\section{REFERENCES}

1. J. A. Asvestas, Integral equations for penetrable electromagnetic scatters, presented as Report RM-857, Grumman Corporate Research Center (June 1987).

2. K. E. Atkinson, Two-grid iteration methods for linear integral equations of the second kind on piecewise smooth surfaces in $\mathscr{R}^{3}$, Rep. Comp. Math. 14 (1991).

3. D. Colton and R. Kress, Integral methods in scattering theory, Wiley, New York, 1983.

4. T. Cwik, Parallel decomposition methods for the solution of electromagnetic scattering problems, Electromagnetics 12 (1992), 343-357.

5. T. Cwik, J. Patterson, and D. Scott, Electromagnetic scattering calculations on the Intel touchstone, presented at Proceedings IEEE Supercomputing (Nov. 1992).

6. A. Edelman, Large dense numerical linear algebra in 1993, presented at UC-Berkeley, preprint, 1992.

7. S. M. Rao, D. R. Wilton, and A. W. Glisson, Electromagnetic scattering by surfaces of arbitrary shape, IEEE Trans. Antennas and Propagation AP-30 (1982), 409-418.

8. $\mathrm{Z}$. Wu and $\mathrm{T}$. Coleman, A parallel row distributed linear algebra system, Technical report CTC92TR93, Cornell University, Ithaca, NY, 1992.

Applied Mathematics Department, The University at Stony Brook, Stony Brook, New YORK 11794

(J. S. Asvestas) Corporate Research Center, Grumman Corporation, Bethpage, New YoRK 11714 